\section{Discontinuity of seen motion reduces the visual motion aftereffect*}

\author{
WILLIAM P. BANKS† and DAN A. KANE \\ Pomona College, Claremont, California 91711
}

Would a motion-picture film of a rotating spiral induce a spiral aftereffect? This question was studied in two experiments in which Ss viewed an animated film of circles collapsing to a point. The rate of apparent motion of the collapsing circles and the discontinuity of motion-the length of jump between successively projected circles-were varied independently. A visual aftereffect like the spiral aftereffect was created. The aftereffect increased in strength and duration with the rate of motion, but at all rates of motion it declined as discontinuity of motion increased. The results are taken as evidence that motion aftereffects are caused by selective fatigue of small, directionally sensitive motion-receptive fields.

A visual motion aftereffect can be induced by viewing a fixed point as a pattern (e.g., a band of stripes) moves uniformly by in a constant direction in the visual field. Virtually any object or pattern seen in the area of the visual field where the inducing motion fell will be subject to the aftereffect. The aftereffect is a paradoxical sort of motion without displacement that is in a direction opposite to that of the inducing motion. When a rotating spiral is used for the inducing motion, the aftereffect is termed the spiral aftereffect, but it is probably based on the same mechanisms as linear motion aftereffects. The spiral aftereffect moves in a direction opposite to the apparent motion of the rotating spiral that induced it. If the moving spiral seemed to collapse inward, the aftereffect is an expanding motion, and vice versa. (Holland, 1965, has a brief but comprehensive review of research on the spiral aftereffect.)

A number of researchers have called attention to the fact that movement aftereffects can be explained elegantly in terms of motion-receptive units in the human visual system (Sutherland, 1961; Barlow \& Hill, 1963; Sekuler \& Pantle, 1967). According to this explanation, there is a large population of motion detectors in the visual system, each serving a small angle of visual field and responding best to motion in a single ("preferred")

*The authors thank W. R. Garner for lending his Perceptiscope and DigiBits programming equipment for the second experiment, R. L. Gottwald and $G$. Felfoldy for assistance with the apparatus, W. Kotef for programming the CALCOMP plotter, and $C$. $K$. Peck, A. Pantle, and D. Berger for advice in the preparation of this article. A Pomona College research grant and summer fellowship to W.P.B. supported most of this work.

tRequests for reprints should be sent to William P. Banks, Department of Psychology, Pomona College, Claremont, California 91711 . direction. Different units have various moderate size is supplied with detectors for every possible direction of motion. When the inducing motion passes across the visual field, it triggers only those detectors responsive to motion in that direction. As the inducing motion is repeated, the units which respond become fatigued. When the motion stops, their firing rates are reduced and remain depressed relative to those of their nonfatigued neighbors for a short period of time. A process central to these units is assumed to compare the outputs of units with various preferred directions and report motion in the direction whose motion-receptive units are most active. Thus, for as long as the units fatigued by the inducing motion fire less often than the unaffected units, the comparative process will signal motion in a direction opposite to that of the inducing pattern, superimposing signals of motion on stationary patterns within the area where the inducing motion fell.

This account of the motion aftereffect has a number of predictions about the parameters of the aftereffect in man beyond those (such as inspection duration, speed of inducing motion, and so forth) usually studied. The prediction examined here is that an inducing motion which is not smooth but has small discrete steps will produce less aftereffect as the size of the steps is increased, even if the discontinuity of motion is not perceptible. This prediction follows from the evidence that motion-receptive fields subtend small visual angles (cf. Hubel \& Wiesel, 1962; Michael, 1968). Progressively increasing the discontinuity of motion should cause more and more of the fields to be skipped, not to be fatigued, and therefore not to contribute to a motion aftereffect.

The present experiments used an preferred directions, and any area of inducing motion which was not continuous but moved in small discrete steps. Animated films of circles with successively decreasing radii were used to induce an aftereffect of the sort a rotating spiral creates. Five films were made by photographing computer-generated (CALCOMP) circles in an animation frame in such a way that each film had a different size of step between radii of successively photographed circles. Five concentric circles, evenly spaced, were photographed on each frame so that the appearance of these films, when viewed on a projection screen, was of five circles collapsing in succession to their common center.

In Experiment 1, Ss viewed all five films at the same projector speed, and it was predicted that the aftereffect would become shorter as discontinuity increased. In Experiment 2, both discontinuity and speed of motion were varied, and it was predicted that increasing the discontinuity would decrease the aftereffect at all speeds of motion.

\section{EXPERIMENT 1} Method

Seven Ss, Pomona College students, viewed all five films at a distance of 72 in. $(184 \mathrm{~cm})$ from a white paper screen on which the films were front-projected at 24 frames/sec. From the Ss' perspective, the stimuli were ellipses with an eccentricity of about 1.1. Along the minor axis, the largest circle subtended $8 \mathrm{deg} 24 \mathrm{~min}$ of the Ss' field, the width of line was $1 \mathrm{~min}$ 1 sec of arc, the smallest jump (of which the others are multiples) was $2 \mathrm{~min} 30 \mathrm{sec}$, and the mean rate of motion of the edges was $1 \mathrm{deg}$ $2 \mathrm{~min} / \mathrm{sec}$. Ss viewed the screen through dark-adaptation goggles (Polaroid nonpolarizing red, XDA8FAP) to reduce the brightness of the image. [Use of these goggles also eliminates the somewhat remote possibility of rod-cone interactions accounting for the aftereffect (Granit, 1928). ] Measured through the goggles, the luminance of the field was about $10 \mathrm{ml}$. Ss adapted to the goggles for $5 \mathrm{~min}$ and then had the aftereffect demonstrated with the film with least discontinuity.

During experimental trials, Ss stared fixedly at the center of the projected display for an inducing time of $2 \mathrm{~min}$ and then shifted their gaze to a sheet of circles from the CALCOMP output placed $5 \mathrm{ft}(160 \mathrm{~cm})$ in front of them. The aftereffect was thus seen as an expansion of the circles on the inspection figure. They timed the aftereffect with a stopwatch. Because the aftereffect seemed to wax and wane, they were instructed to let the watch run while they saw the 


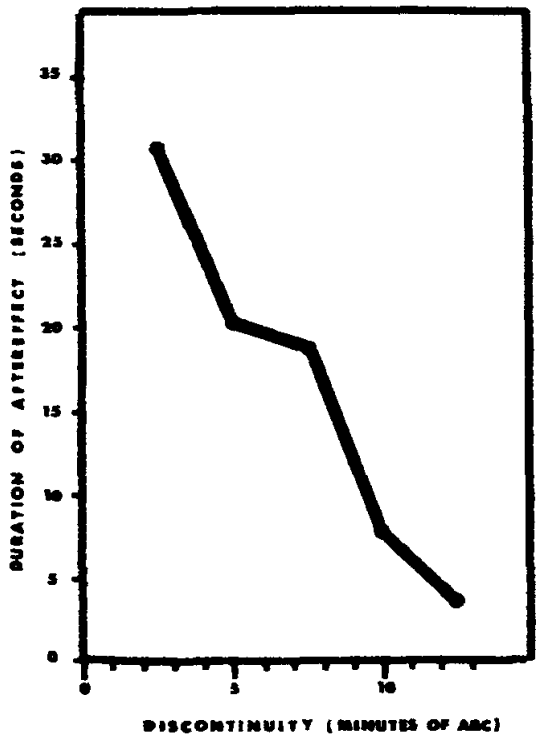

Fig. 1. Mean duration of motion aftereffect as a function of discontinuity of inducing motion after 2 min of viewing.

aftereffect and to stop it when they did not. When the aftereffect had finally dissipated, the $E$ recorded the stopwatch reading and waited $5 \mathrm{~min}$ to allow recovery from the aftereffect before showing the next inducing stimulus. Initially, five Ss adapted once to each film in an order counterbalanced for position. The position of the film in the inspection series seemed to have no effect, and data from two additional Ss who saw the films in random order were added.

\section{Results}

The mean duration of aftereffect for the seven $S_{s}$ is plotted as a function of discontinuity in Fig. 1 . The predicted reduction in duration of aftereffect is strong and significant, with a $F(4,24)$ of $41.6(p<.001)$.

It was observed that the more discontinuous circles appear to be moving more slowly than the more continuous ones when shown at the same projector speed. The second experiment, in which speed as well as discontinuity of inducing motion was varied, eliminates the possibility that these apparent speed differences account for the differences in duration of aftereffect.

\section{EXPERIMENT 2 \\ Method}

In this experiment, only the four films with the least discontinuity were used. Four new Ss viewed these on a high-contrast Kodak screen at a number of different projector speeds. Viewing distance was 96 in. $(246 \mathrm{~cm})$. The largest circle subtended $9 \mathrm{deg}$
$32 \mathrm{~min}$ of arc, line thickness was $1 \mathrm{~min}$, and the smallest jump was $2 \mathrm{~min} 52 \mathrm{sec}$. No goggles were used, and the luminance of the image was about $8 \mathrm{~mL}$. Ss warmed up with two adaptation periods of $1 \mathrm{~min}$ to the second film (discontinuity $5 \mathrm{~min}$ $43 \mathrm{sec}$ ), moving at $1 \mathrm{deg} 12 \mathrm{~min} / \mathrm{sec}$. During experimental trials, each $\mathbf{S}$ was presented every combination of film and speed for $30 \mathrm{sec}$ in an irregular order counterbalanced over Ss. After each inducing stimulus was viewed for the $30 \mathrm{sec}$, the projector was stopped, leaving a frame of stationary circles projected on the screen. Ss gave magnitude estimations of the strength of the aftereffect (Sekuler \& Pantle, 1967, used magnitude estimation similarly, but had Ss judge the apparent velocity of the aftereffect) immediately and timed its duration with a stopwatch. "Strength" was defined for the $S s$ as the phenomenal clarity and "noticeableness" of the aftereffect. These judgments are probably correlated with apparent velocity of the aftereffect, but the aftereffects of some of the stimuli were so weak that apparent velocity judgments would have been difficult to make. Two minutes were allowed for recovery from the aftereffect of each film before the next was shown, and a 7-min rest period was given each $S$ in the middle of the series.

\section{Results}

Figure 2 shows how the duration of

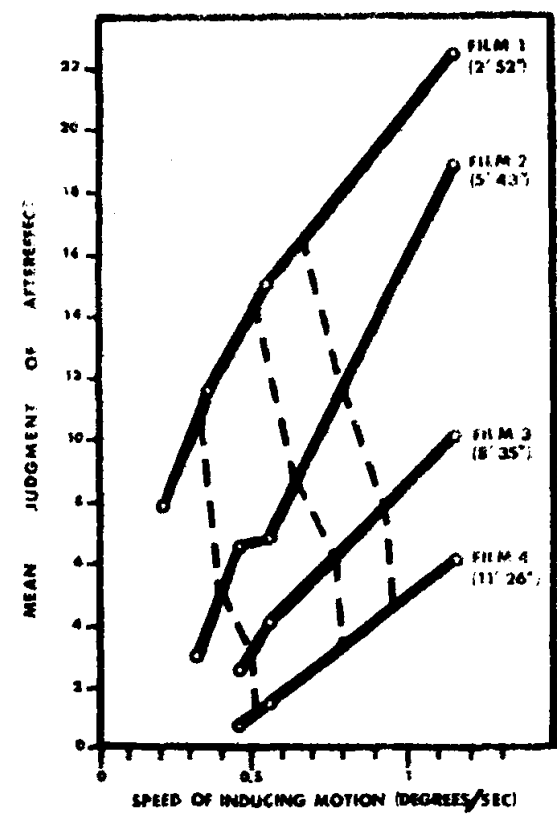

Fig. 3. Mean judgment of strength of motion aftereffect as a function of speed for four levels of discontinuity of inducing motion. Parameters and dashed lines are the same as in Fig. 2

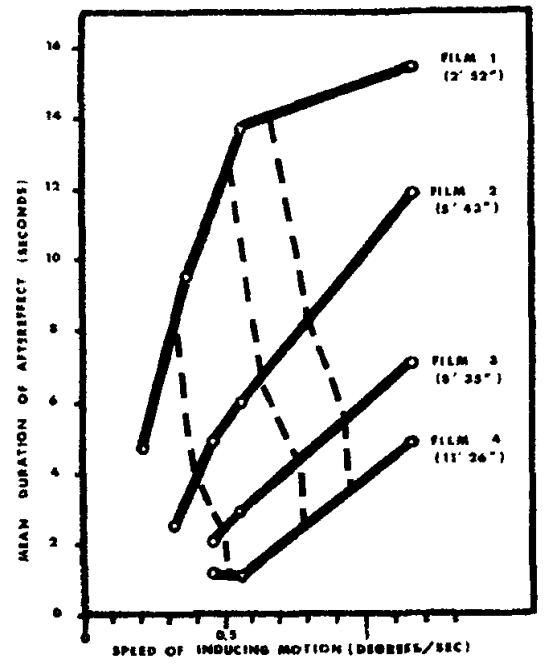

Fig. 2. Mean duration of motion aftereffect as a function of inducing speed for four levels of discontinuity of inducing motion. Length of jump between successive steps of discontinuous motion is in parentheses below each film designation. Viewing time was 30 sec. Dashed lines connect points of equal apparent inducing speed.

aftereffect varied with speed for each film, and Fig. 3 shows how the judged strength of aftereffect varied. The duration of aftereffect for $1 \mathrm{deg}$ $2 \mathrm{~min} / \mathrm{sec}$ estimated from Fig. 2 is, at each level of discontinuity, about half that found in Experiment 1 (where inducing speed was $1 \mathrm{deg} 2 \mathrm{~min} / \mathrm{sec}$ ). This difference between the experiments probably results from the fact that the inducing time was $2 \mathrm{~min}$ in Experiment 1 and $30 \mathrm{sec}$ in Experiment 2, but other differences between the two experimental procedures (i.e., recovery period, luminance, goggles) may have contributed to the differences in duration of aftereffect.

The dashed lines in these figures connect points for which the apparent speeds of the inducing stimuli are equal. These equal apparent speed contours were derived from a previous scaling experiment in which the same four Ss gave magnitude estimations of the apparent speed of each film at various projector speeds. If the aftereffect functions in Figs. 2 and 3 were plotted as a function of apparent inducing speed, these contours would be vertical. Since the contours are quite steep, rectifying the aftereffect functions according to the apparent inducing speed contours (i.e., using apparent rather than actual speed as the abscissa) would not reduce the effect of discontinuity very much.

An analysis of variance was performed only on the duration 
measures (the strength judgments are highly correlated with duration). Data from two combinations of discontinuity and speed (Film 1 at $0.2 \mathrm{deg} / \mathrm{sec}$ and Film 2 at $0.3 \mathrm{deg} / \mathrm{sec}$ ) were not included in the analysis so that an orthogonal design could be used. The remaining data were cast into a three-factor 4 (discontinuity) by 3 (speed) by 4 (Ss) design. The main effects of discontinuity and speed were tested against pooled error terms because neither factor interacted significantly with Ss. The main effects of both discontinuity and speed were significant beyond the .01 level, with an $F(3,27)$ of 23.74 and an $F(2,24)$ of 19.72 , respectively. (Use of an unpooled $\mathbf{S s}_{s}$ by Treatment error term results in the same level of significance of the main effects.)

\section{DISCUSSION}

As discontinuity of motion increases, the aftereffect decreases. This fact is taken as evidence for a motion-detecting system that gathers information from $8 \mathrm{mall}$ motion-receptive fields, but there are other ways of interpreting the results, some of them compatible with the receptive field notion.

One intriguing interpretation is suggested by Gregory's (1964, 1966) hypothesis of two motion-detecting systems in the visual apparatus. One of these systems, the positional system, detects motion by comparing successive positions of an object or by comparing the position of one object with another or with the background. This system would, presumably, be responsible for the phi phenomenon and some illusions of relative motion. The other system hypothesized by Gregory is the velocity system. This system gives direct signals of motion, probably by interpreting local displacements of contours on the retina. As Gregory has pointed out, motion aftereffects seem to be a disturbance of the velocity system and not the positional system, since the aftereffect has illusory motion but not illusory displacement. An interpretation of the present study in terms of the two systems of motion detection is straightforward: Changes in discontinuity of motion affect the two systems differentially. The velocity system seems to be greatly affected by discontinuity because the aftereffect, mediated by the velocity system, drops off rapidly as discontinuity increases. The positional system seems less sensitive to discontinuity of motion because the apparent motion of the inspection display, presumably mediated largely by the positional system, is not much affected by discontinuity of motion within the limits of discontinuity used here. It seems reasonable to conclude, further, that the velocity system depends on receptive fields to detect motion and that the positional system uses a different mechanism.

Lichtenstein (1963) used an ingenious system to present discontinuously moving lines on the face of an oscilloscope. He studied sensitivity to rather than the aftereffect of discontinuous motion, but some of his observations are of interest here. First, he found movement sensitivity to be proportional to the "space-time density of the stimuli producing the motion." Figures 2 and 3 show that speed and discontinuity can be traded for each other and thus that the aftereffect may also be proportional to the space-time density of the inducing stimulus. Considerably more precise and complete functions would, however, be necessary before this conclusion could be drawn with any confidence. Second, Lichtenstein found that with high stimulus velocities and peripheral viewing, the oscilloscope traces could sometimes not be seen clearly but had a considerable apparent rate. On the basis of this and other observations, he suggested that motion may be perceived without a perception of a thing moving. Such a possibility is expected if motion is detected by receptive fields. A field could be triggered by motion whether or not a moving object was perceived.

It was hoped that the form of the discontinuity effect in the present study could be used to estimate the average size and other properties of the motion-receptive fields. Unfortunately, these estimates cannot be made without a precise model of the properties and arrangement of the fields, and no such model presently exists (but see Thomas, 1970). The results do seem to indicate that the fields are fairly small, since the aftereffect all but disappears by the time discontinuity has reached $10 \mathrm{~min}$ of arc. This small estimated size of the fields might seem to be in conflict with the fact that motion-responsive units in physiological preparations rarely serve less than .5-1 deg of arc. It should be pointed out, however, that a field represented even at the retinal ganglion level must contain many smaller units; otherwise, the response would not be maintained continuously over the field. Thus, the discontinuity effect would seem to be largely a result of the smaller units within receptive fields being "skipped" and not being fatigued by the discontinuous motion. Barlow and Levick (1965) have proposed a model of the neural organization within the motion receptive fields of the rabbit's retina that relies on interconnections among smaller subunits. Their experimental estimates of the size of these subunits placed them at between 15 and $30 \mathrm{~min}$ of visual angle. The results of the present experiments would be in agreement with approximately the same estimate of the size of subunits in human receptive fields.

Finally, it must be stated that the question of where motion-receptive units are situated in the visual system is not answered by this research, nor was it asked in the first place. This study demonstrates what might be termed a granularity in motion perception, or, at least, in one component of it. The effect of discontinuity was discussed in terms of motion-receptive units of cells being skipped, but the units could be anywhere. The cells are surely retinal, but the neural organization that combines them to form a unit may or may not involve structures deeper than the retina. It might, however, be possible to use the discontinuity effect to discover whether or not the units were retinal. If thecollapsing circles of this experiment were presented binocularly to alternate eyes, such that the first circle in the series was presented to the left eye, the next to the right, the next to the left, and so on, and if the discontinuity effect were the same as that with presentation of all circles to a single eye, the units would have to be central. If the aftereffect in such a situation were the same as that found with twice the discontinuity presented monocularly, the units would have to be retinal, or at least specific to each retina.

\section{REFERENCES}

BARLOW, H. B. HILL, R. M. Evidence for a physiolotical explanation of the waterfall phenomenon and tigural after-effects. Nature, $1963,200$. 1345-1347.

BARLOW, H. B. \& LEVICK, $\mathbf{w}$. R. The mechanism of directionally selective units in rabbit's retina. Journal of Physiology, $1965,178,477-504$.

GRANIT, $R$. On inhibition in the after-effect of seen movement. British Journal of Psychology, 1928, 19. 147-167.

GREGORY, R. L. Human perception British Medical Bulletin. 1964, 20,21-26.

GREGORY, R. L. Eye and brain. New York: McGraw-Hill, 1966.

HOLLAND, H. C. The spiral after-effect. New York: Pergamon Press, 1965.

HUBEL, D. H., \& WIESEL, T. N. Receptive fields, binocular interaction, and functional architecture in the cat's visual cortex. Journal of Physiology, 1962, 160 , 106-154.

LICHTENSTEIN, M. Spatio-temporal factors in cessation of smooth apparent motion. Journal of the Optical Society of America, 1963, 53, 304-306.

MICHAEL, C. R. Receptive fields of single optic nerve fibers in a mammal with an all-cone retina. Journal of Neurophysiology, 1968, 31, 257-267. 
SEKULER, R. W.. \& PANTLE, A. A model for after-effects of seen movement. Vision Research, 1967, 7, 427-439.

SUTHERLAND, $\dot{N}$ S. Figural after-effects and apparent size. Quarterly Journal of Experimental Psychology, 1961, 13, 222-228.

THOMAS, J. P. Model of the function of receptive fields in human vision. Psychological Review, 1970, 77, 121-134. (Accepted for publication February 15, 1972.) 